\title{
The dark matter halos of spheroidal galaxies and clusters of galaxies
}

\author{
Tommaso Treu \\ California Institute of Technology, Astronomy 105-24, Pasadena CA \\ 91125; present address: University of California at Los Angeles, \\ Astronomy 6 Astrophysics, Los Angeles, CA 90095 \\ Léon V. E. Koopmans \\ Space Telescope Science Institute, 3700 San Martin Dr, Baltimore MD \\ 21218 \\ David J. Sand, Graham P. Smith, Richard S. Ellis \\ California Institute of Technology, Astronomy 105-24, Pasadena CA \\ 91125
}

\begin{abstract}
We describe the first results from two observational projects aimed at measuring the amount and spatial distribution of dark matter in distant early-type galaxies (E/S0s) and clusters of galaxies. At the galaxy scale, the Lenses Structure and Dynamics (LSD) Survey is gathering kinematic data for distant (up to $z \sim 1$ ) E/S0s that are gravitational lenses. A joint lensing and dynamical analysis constrains the fraction of dark matter within the Einstein radius, the mass-to-light ratio of the stellar component, and the total slope of the mass density profile. These properties and their evolution with redshift are briefly discussed in terms of the formation and evolution of E/S0 galaxies and measurement of the Hubble Constant from gravitational time delay systems. At the cluster scale - after careful removal of the stellar component with a joint lensing and dynamical analysis - systems with giant radial arcs can be used to measure precisely the inner slope of the dark matter halo. An HST search for radial arcs and the analysis of a first sample are briefly discussed in terms of the universal dark matter halos predicted by CDM simulations.
\end{abstract}

\section{Introduction}

Decades after the discovery of dark matter around spiral galaxies little is known about dark matter in early-type galaxies. Mass tracers at large radii (such as stellar kinematics, kinematics of globular clusters and of planetary nebulae, and $\mathrm{X}$-ray halos) generally indicate that a constant mass-to-light ratio cannot reproduce the observations, although there are typically large uncertainties and a wide variety of behavior is seen. The main source of uncertainty in interpreting kinematic measures is that the derived mass profile depends on the assumed orbital structure. This is commonly referred to as the mass-anisotropy degeneracy. 
The situation is even more uncertain outside the local Universe, because traditional kinematic tracers at large radii are generally not feasible at cosmological distances. However, additional constraints can be gathered by looking at early-type galaxies that are gravitational lenses. The configuration of multiple images provides information on the mass distribution of the lens; first and foremost a robust measurement of the mass enclosed within the Einstein Radius (typically larger than the effective radius), independent of its kinematic status.

The Lenses Structure and Dynamics (LSD) Survey (Koopmans \& Treu 2002, 2003; Treu \& Koopmans 2002, 2003; hereafter KT) is obtaining internal kinematics for a sample of of 11 relatively isolated E/S0 lens galaxies at $z=0.04-1.01$. A joint lensing and dynamical analysis is used to break the mass-anisotropy degeneracy and determine the amount and distribution of luminous and dark matter. The first results from this project are briefly summarized and discussed in Section 2.

At the cluster scale, the presence of dark matter has been known for a long time (Zwicky 1937). The distribution of dark matter has been probed over a wide range of scales using X-ray analysis, dynamical studies and gravitational lensing, showing that dark matter dominates in mass. The wealth of mass tracers in clusters - in particular the existence of giant radial arcs - makes them an ideal laboratory to test the existence of universal mass density profiles predicted by cold dark matter (CDM) simulations. Combining a lensing analysis of clusters with giant arcs with a dynamical analysis of the kinematics of the brightest cluster galaxy (BCG), we can measure very accurately the mass density profile within $\sim 100 \mathrm{kpc}$, disentangle the stellar and dark matter, and pinpoint the inner logarithmic slope of the dark matter halo. This has motivated a search of suitable systems with a central dominant BCG and giant arcs through the entire HST-WFPC2 archive (Sand et al. 2004, in preparation), and the spectroscopic follow-up at Keck (Sand, Treu \& Ellis 2002; Sand et al. 2003). The first results from this project are briefly summarized and discussed in Section 3.

\section{Early-type galaxies: dark halos and cosmic evolution}

Figure 1 illustrates via the lens 0047-281 ( $z=0.485$; Warren et al. 1996) the data we are collecting as part of the LSD Survey. Archival HST imaging data (mostly from the CASTLeS collaboration) are combined with stellar kinematic data obtained using ESI at the Keck-II Telescope. In excellent conditions (seeing $\left.\sim 0.6^{\prime \prime}\right)$ it is possible to obtain a spatially resolved stellar velocity dispersion profile (hatched boxes in the right panel) out to the Einstein Radius (typically 1-2 effective radii, 1-2 arcseconds). A joint lensing and dynamical analysis is used to constrain the free parameters of a family of two-component mass models. One component represents the stellar mass and follows the HST luminosity profile scaled by a stellar mass-to-light ratio, the other component represents the dark matter halo and is modeled as a generalized Navarro, Frenk \& White (1997) profile (see KT for details).

The main results from analysis of the first two objects (0047-281 and MG2016) are: 

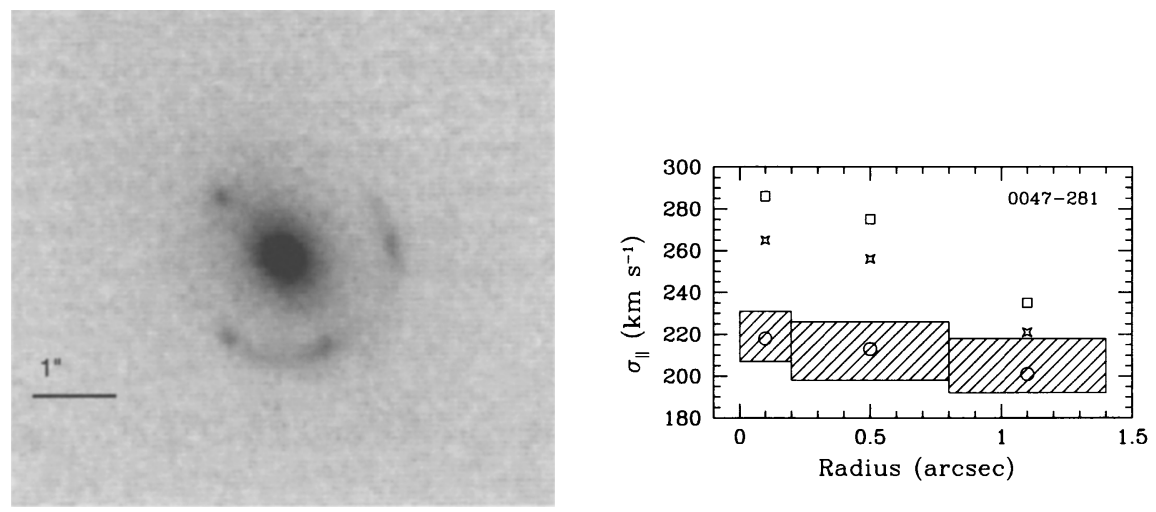

Figure 1. Left: HST image of $0047-281$ at $z=0.485$. Right: velocity dispersion profile of $0047-281$ along the major axis. The box height indicates the $68 \%$ measurement error, whereas the box width indicates the spectroscopic aperture. The open squares are the corresponding values for an isotropic constant $M / L$ model, which is rejected by the data. See Koopmans \& Treu (2003) for details.

1. Extended dark matter halos are detected in high redshift E/S0 galaxies at high significance $(>10 \sigma)$; dark matter contributes $50-75 \%$ of the total mass within the Einstein radius.

2. The evolution of the stellar mass-to-light ratio with redshift, obtained from a two-component dynamical model, agrees with the independent estimate obtained by studying the evolution of the Fundamental Plane with redshift (Treu et al. 2002; van Dokkum \& Ellis, Gebhardt et al. 2003). This is consistent with no structural or dynamical evolution of E/S0s between $z=1$ and today.

3. The effective slope (i.e. the average slope measured between roughly the effective and Einstein radius) of the total mass distribution is very close to isothermal, i. e. $\rho \propto r^{-\gamma^{\prime}}$ with $\gamma^{\prime} \sim 2.0$ (to within 5\%), which we interpret as a possible indication of (incomplete) violent relaxation. This result, if confirmed by a larger sample, indicates that already at $z \sim 1$ there is a mechanism by which dark and luminous component conspire with each other to produce almost perfectly flat rotation curves, while still preserving their spatial segregation.

Clearly, analysis of the rest of the sample is essential to confirm and extend these findings. For example, an open question - which we intend to address and has profound cosmological implications - is understanding what is the distribution of the effective slope of the total mass density profile. An intrinsic scatter is expected on the basis of local studies (e.g. Gerhard et al. 2001). It is necessary to quantify this scatter in order to estimate the uncertainties in the determination of the cosmological parameters from lens statistics, of velocity dispersion of early-type galaxies from image separation, and of the Hubble Constant from gravitational time delays. The Hubble Constant is highly sensitive to the slope of the mass density profile, and some external information is needed if one wants to measure it with precision better than a few tens of per cent. In an effort parallel to the LSD Survey, an enlarged collaboration is targeting lenses with known 
time-delays for spectroscopic follow-up with Keck and VLT. Although this is generally complicated by the presence of bright QSO images outshining the lens galaxy, analysis of the first two systems (Treu \& Koopmans 2002; Koopmans et al. 2003) confirms that with suitable kinematic data it is possible to measure $\mathrm{H}_{0}$ at $\sim 15 \%$ accuracy from each individual system, including the uncertainty on the mass density profile. We are collecting data for more systems, with the goal of measuring $\mathrm{H}_{0}$ from time-delays with precision comparable to the HST Key Project (Freedman et al. 2001).

\section{Galaxy clusters: the inner density profile and the CDM "cusp" problem}

As part of our study of the inner regions of clusters of galaxies we have measured arc redshifts and BCG stellar velocity dispersions for 6 clusters of galaxies (Figure 2; Sand et al. 2002, 2003). Three systems (upper panels) are selected to have both a radial and a tangential gravitational arc, while three systems (lower panels) are selected to have only tangential arcs. The location of the symmetry break in a radial arc constrains the derivative of the enclosed mass and therefore the radial arc systems provide very tight constraints on the shape of the mass density profile. However, the radial critical line disappears when the total mass density profile becomes steeper than $r^{-2}$. Therefore a radial arc selected sample may possibly be biased toward flatter mass profiles. For this reason we introduced the tangential arc only systems as a control sample.
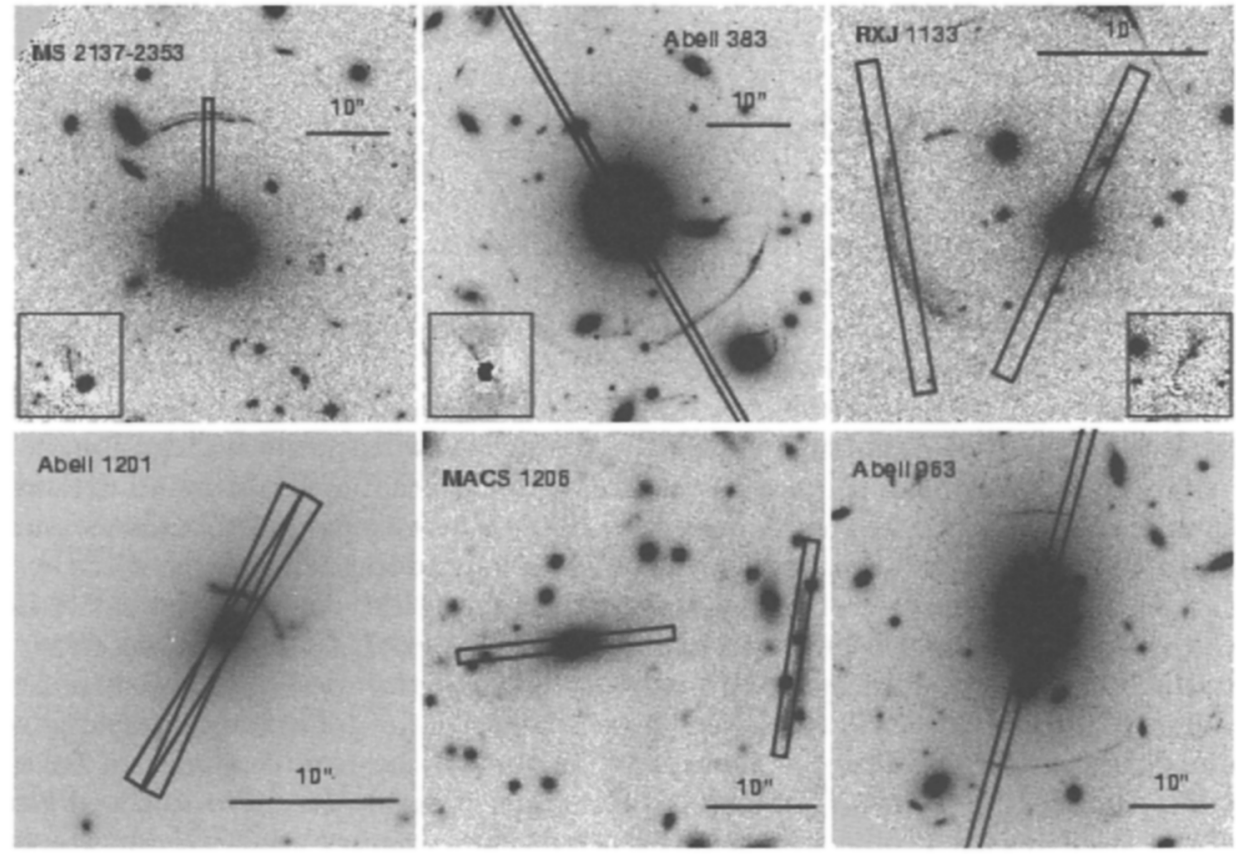

Figure 2. Images of the six clusters studied by Sand et al. (2003) 
A joint lensing and dynamical analysis is performed, fitting a family of two component mass models to the data (see Sand et al. 2003 for details). Specifically, the dark matter mass component is parametrized as a generalized NFW profile (Navarro, Frenk \& White 1997; Moore et al. 1998), i.e. the dark matter density goes as $r^{-\beta}$ at small radii and $r^{-3}$ at large radii. Numerical simulations predict $\beta$ in the range 1-1.5 (hereafter, respectively, NFW and Moore slopes), although numerical issues are still being debated (see Sand et al. 2003 and references therein).
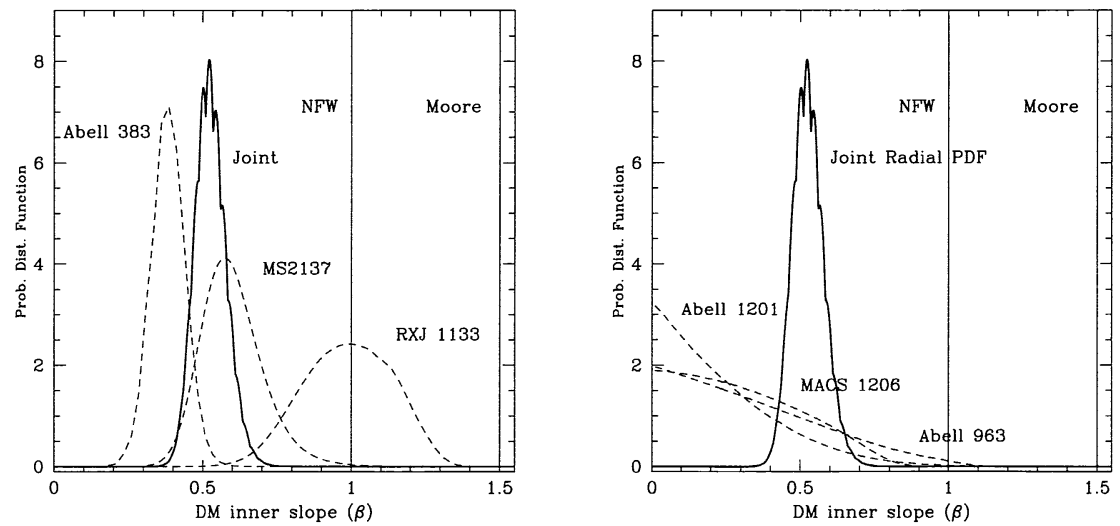

Figure 3. Left: Probability distribution function of the DM inner density slope, $\beta$, for the three radial arc clusters. Right: Probability distribution function of the DM inner density slope, $\beta$, for the tangential arc sample. Also plotted is the joint PDF for the radial arc sample and the tangential arc sample. (from Sand et al. 2003)

Figure 3 shows the posterior probability density for $\beta$ for the radial (left) and tangential (right) arc samples. The radial arc sample indicates generally flat inner slopes, the average slope being inconsistent with the CDM "cusps" at more than $99.99 \%$ CL. However, intrinsic scatter is detected and individual systems can be as steep as NFW (RXJ1133). The tangential arc systems also confirm flatter slopes than $\beta=1$, indicating that the sample with radial arcs is unbiased.

The implications of this discrepancy could be profound. Either the CDMonly simulations are insufficiently accurate and/or contain insufficient physics to describe faithfully the inner regions of clusters. For example, baryons could play an important dynamical role driving energy and angular momentum out of the cluster core through mechanisms such as dynamical friction (e.g. El-Zant et al. 2003). Alternatively, the DM could be partially self-interacting, or manifest some interaction with the baryons (Spergel \& Steinhardt 2000). Either outcome would be very exciting. On the one hand, numerical simulations are being developed which will permit the realistic inclusion of baryons. Finding mechanisms that satisfy our observational constraints will be invaluable in understanding the complex physics of star formation and dissipative processes. And of course, 
there is the possibility that there is some fundamental flaw in CDM which will necessitate considering more exotic scenarios, like self-interacting DM.

We are currently looking to expand our sample, drawing from the candidate radial arc systems identified from the HST-WFPC2 archive (Sand et al. 2004). This would improve our determination of the mean of $\beta$ and also provide a first measure of the intrinsic scatter, which is essential for any meaningful comparison with simulations, especially those that include baryons.

\section{Acknowledgments}

TT thanks the organizers for this pleasant and exciting meeting. TT thanks Eric Agol, Stefano Borgani, Amr El-Zant, Raul Jimenez, Masataka Fukugita, Jean-Paul Kneib, Ben Moore, Joel Primack, Paul Schechter, Jerry Sellwood and Simon White for stimulating conversations, during and around the Sydney Meeting. TT gratefully acknowledges support from an AAS travel grant and from HST proposals AR-09222, AR-09527, AR-09960, provided by NASA through a grant from STScI, which is operated by AURA, Incorporated, under NASA contract NAS5-26555.

\section{References}

El-Zant, A. et al. 2003, ApJ, submitted, astro-ph/0309412

Freedman, W. et al. 2001, ApJ, 553, 47

Gebhardt, K. et al. 2003, ApJ, in press, astro-ph/0307242

Gerhard, O., Kronawitter, A., Saglia, R. P., \& Bender, R. 2001, AJ, 121, 1936

Koopmans, L.V.E. \& Treu, T. 2002, ApJ, 568, L5

Koopmans, L.V.E. \& Treu, T. 2003, ApJ, 583, 606

Koopmans, L.V.E., Treu, T., Fassnacht, C.D., Blandford, R.D. \& Surpi G. 2003, ApJ, 599, in press

Moore, B., Governato, F., Quinn, T., Stadel, J. \& Lake, G. 1998, ApJ, 499, L5

Navarro, J., Frenk, C. S., \& White S. D. M 1997, ApJ, 490, 493

Sand, D.J., Treu, T. \& Ellis R.S. 2002, ApJ, 574, L129

Sand, D.J., Treu, T., Smith, G.P. \& Ellis, R.S. 2003, ApJ, submitted, astro$\mathrm{ph} / 0309465$

Spergel, D. \& Steinhardt, P. 2000, Phys. Rev. Lett., 84, 3760

Treu, T. \& Koopmans, L.V.E. 2002, ApJ, 575, 87

Treu, T. \& Koopmans, L.V.E. 2003, MNRAS, 343, L29

Treu, T., Stiavelli, M., Casertano, S., Møller, P. \& Bertin 2002, ApJ, 564, L13 van Dokkum, P.G. \& Ellis, R.S. 2003, ApJ, 592, L53

Warren, S.J., Hewett, P.C., Lewis, G.F., Møller, P., Iovino, A., Shaver, P.A. 1996, MNRAS, 278, 139

Zwicky, F. 1937, ApJ, 82, 217 